\title{
Energy absorption capacities of kenaf fibre-reinforced epoxy composite elliptical cones with circumferential holes
}

\begin{abstract}
In the present study, the influence of circumferential holes on the energy absorption capacity and load carrying ability of kenaf fibre-reinforced composite elliptic tubes was experimentally investigated. A series of experiments were performed for composite elliptical tubes with different circumferential perforations ( $0,4,6$ and 8 holes). This range is suitable for obtaining the adjusted distance. Kenaf fibre matte foam was used in this study due to several advantages, such as low cost, low health risk, light weight, and availability. The kenaf was $4 \mathrm{~mm}$ thick with a density of $0.17 \mathrm{~g} / \mathrm{cm}^{3}$. To investigate the influence of circumferential holes on the energy absorption capability, a pseudo static axial crash test was independently performed on these samples. The results showed that the structure failed in progressive failure mode and showed crushing failure with longitudinal cracks. The test results revealed that the specific energy assimilated by the compound elliptical tube with 8 circumferential holes showed the highest mean crushing stress value compared with other compound elliptical tubes.
\end{abstract}

Keyword: Axial crush; Circumferential holes; Elliptical cones; Energy absorption; Kenaf composites 